\title{
Magnetic oscillations measure interlayer coupling in cuprate superconductors
}

\author{
P.D. Grigoriev ${ }^{1,2,3}$ and Timothy $\operatorname{Ziman}^{4,5}$ \\ ${ }^{1}$ L. D. Landau Institute for Theoretical Physics, 142432 Chernogolovka, Russia \\ ${ }^{2}$ National University of Science and Technology "MISiS", Moscow 119049, Russia \\ ${ }^{3}$ P.N. Lebedev Physical Institute, RAS, 119991, Moscow, Russia \\ ${ }^{4}$ Institut Laue-Langevin, BP 156, 41 Avenue des Martyrs, 38042 Grenoble Cedex 9, France \\ ${ }^{5}$ LPMMC (UMR 5493), Université de Grenobles-Alpes and CNRS, \\ Maison des Magistères, BP 166, 38042 Grenoble Cedex 9, France
}

(Dated: August 23, 2021)

\begin{abstract}
The magnetic oscillations in YBCO high-temperature superconductors have been widely studied over the last decade and consist of three equidistant low frequencies with a central frequency several times more intense than its two shoulders. This remains a puzzle in spite of numerous attempts to explain the corresponding small Fermi-surface pockets. Furthermore the ARPES data indicate only four Fermi-arcs with bilayer splitting, and show no sign of such small areas in the Fermi surface. Here we argue that the magnetic oscillations measured in under-doped bilayer high temperature superconductors, in particular $\mathrm{YBa}_{2} \mathrm{Cu}_{3} \mathrm{O}_{6+\delta}$, provide a measure of the interplanar electronic coupling rather than the areas of fine-grain reconstruction of the Fermi surfaces coming from induced charge density waves. This identification is based on the relative intensities of the different peaks, as well as their angular dependence, which points to an effective Fermi surface that is larger than the oscillation frequencies, and is compatible with several indications from ARPES. The dominance of such frequencies with respect to the fundamental frequencies from the Fermi surface is natural for a strongly correlated quasi-two dimensional electronic systems where non-linear mixings of frequencies are more resistant to sample inhomogeneity.
\end{abstract}

PACS numbers: 74.72.-h,72.15.Gd,73.43.Qt,74.70.-b,71.45.Lr

\section{INTRODUCTION}

Magnetic quantum oscillations (MQO) is a traditional and powerful tool to study electronic structure of various metals $\underline{\underline{1}} \underline{\underline{3}}$ The first observation of MQO in cuprate high-temperature superconductors about a decade ago, $\underline{\underline{4}}$ was rather a surprise given that these are normally taken as indicating a Fermi surface of a normal metallic state. Since then, MQO have been used extensively to investigate the electronic structure in cuprates, both holedoped $^{\underline{5}-\underline{\underline{8}}}$ and electron-doped, $\underline{\underline{9}} \underline{\underline{12}}$ as well as in various Fe-based superconductors $\stackrel{13-19}{-12}$ Probably the most striking data are for the underdoped yttrium barium copper oxide (YBCO) compounds $\mathrm{YBa}_{2} \mathrm{Cu}_{3} \mathrm{O}_{6+\delta}(\underline{4}, 20-27$, reviewed in ${ }^{5-\underline{8}}$, where there is one prominent oscillation peak at frequency $F_{\alpha} \approx 530 T$ with two smaller shoulders at $F_{ \pm}=F_{\alpha} \pm \Delta F_{\alpha}$, where $\Delta F_{\alpha} \approx 90 T$. All three frequencies in YBCO are much smaller than expected from closed pockets of any Fermi surface, seen, for example from ARPES experiments28,29.

The completely unreconstructed Fermi surface of YBCO would consist of one large pocket, almost a square with smoothed corners, filling about one half of the Brillouin zone and corresponding to a large frequency $\sim 10^{4}$ tesla. Fermi-surface reconstruction, possibly caused by the pseudogap, AFM or CDW order, takes place for doping level $p<15 \%$, resulting to four Fermi arcs, as suggested by ARPES 28,29 and schematically shown in Fig. 1b. The scattering by the AFM wave vector $Q=(\pi / a, \pi / b)$, connecting the ends of Fermi arcs, forms closed FS pockets of area about $6 \%$ of the Brillouin zone, corresponding to MQO frequency about 1.6kTesla. The observed magnetic oscillation frequency $F_{\alpha} \approx 530 T$ corresponds to a Fermi-surface cross-section of only $2 \%$ of the Brillouin zone, much less than the size of the pockets suggested by ARPES, without entering into considerations such as whether the "Fermi arcs" can actually be closed. Thus there is a clear inconsistency between the ARPES and MQO experimental data. An unusual alternative source of oscillations was proposed in terms of Andreev-type bound states $\stackrel{30}{=}$ but the predicted change in oscillation frequencies with superconducting gap contradicts experiment.

The situation has been complicated by the subsequent evidence of at least fluctuating and short range charge order in low fields by X-ray scattering, $\stackrel{31-34}{=}$ nuclear magnetic resonance $\mathrm{e}^{35,36}$, and sound velocity measurements 37 . In the high magnetic fields corresponding to the range where magnetic oscillations are observed, superconductivity is gradually suppressed and charge density wave coherence is stabilized $\stackrel{34}{=}$ The measured Hall and Seebeck coefficients $38-\underline{40}$, treated by the simplified theory without taking into account magnetic breakdown, strong electronic correlations and superconducting flux flow contribution, support additional Fermi-surface reconstruction. Thus it is tempting, but we argue misleading, to attempt to explain the observed low-frequency magnetic oscillations by the appearance of new Fermi surface pockets coming from reconstruction of the larger Fermi surfaces by charge density wave order. Different attempts in this direction vary in details such as inclusion of spin-orbit or Zeeman splittings $\stackrel{41-45}{=}$ but it is hard to explain the observed three-peak frequency pattern of quantum oscilla- 
tions without predicting additional frequencies of similar amplitudes from the charge order. Note that a frequency pattern somewhat similar to that of $\mathrm{YBa}_{2} \mathrm{Cu}_{3} \mathrm{O}_{6+\delta}$ is observed in the closely related stoichiometric compound $\mathrm{YBa}_{2} \mathrm{Cu}_{4} \mathrm{O}_{8} \stackrel{46-48}{\underline{4}}$ where there is no indication of a static superstructure. Furthermore if very small Fermi-surface pockets really are the origin of the observed $F_{\alpha}, F_{+}$, and $F_{-}$frequencies, they should depend strongly on doping, which does not seem to be the case: for instance $F_{\alpha}$ changes by only $10 \%$ when the doping $p$ almost doubles, from 0.09 to $0.14 . \underline{\underline{50}}$

In this paper, we argue that the magnetic oscillations in these under-doped bilayer high temperature superconductors in fact provide a measure of the inter-planar electronic coupling and do not, contrary to widespread belief, correspond to areas of fine-grain reconstruction of the Fermi surfaces coming from induced charge density waves. This identification is based on the relative intensities of the different peaks, as well as their angular dependence, which points to an effective Fermi surface that is larger than the oscillation frequencies, and is compatible with indications from ARPES as to the fundamental frequency as well as the bilayer splitting. The dominance of such frequencies with respect to the standard frequencies from the Fermi surface, whose current observation is still somewhat controversial, is natural because the non-linear mixings of frequencies better survive sample inhomogeneity, as we show in detail below.

\section{MAGNETIC OSCILLATION PRODUCED BY INTERLAYER HOPPING}

\section{A. Qualitative idea}

A clue to the origin of the observed tiny MQO frequencies comes from what are called "slow oscillations" (SlO) in organic superconductors 51 . In these quasi-two dimensional compounds, oscillations can be clearly attributed, after no little debate, not to new small pockets of the Fermi surface, but to the mixing of two close frequencies $F_{\beta} \pm \Delta F$, where only the $F_{\beta}$ frequency corresponds to a Fermi-surface area and the frequency splitting $\Delta F$ is due to Fermi-surface warping, whose origin is the interlayer electron transfer integral $t_{z} \underline{\underline{51}}$ Magnetoresistance $(\mathrm{MR})$ oscillations with a much lower beat frequency $F_{\text {slow }}=2 \Delta F=4 t_{z} B / \hbar \omega_{c}$ then arise. What is surprising at first sight is that the amplitude of such emergent slow oscillations is much higher than those of the oscillations with the original frequencies $F_{\beta} \pm \Delta F$, because they are damped neither by temperature nor by long-range disorder (spatial inhomogeneity, leading to variations of $E_{F}$ along the sample on the scale much larger than magnetic length) $)^{51,52}$ (see Sec. IID below). Even in high-quality monocrystals of organic metals such long-range disorder has been shown to make the major contribution to the Dingle temperature .51 In the notoriously inhomogeneous cuprates, such disorder is undoubt-

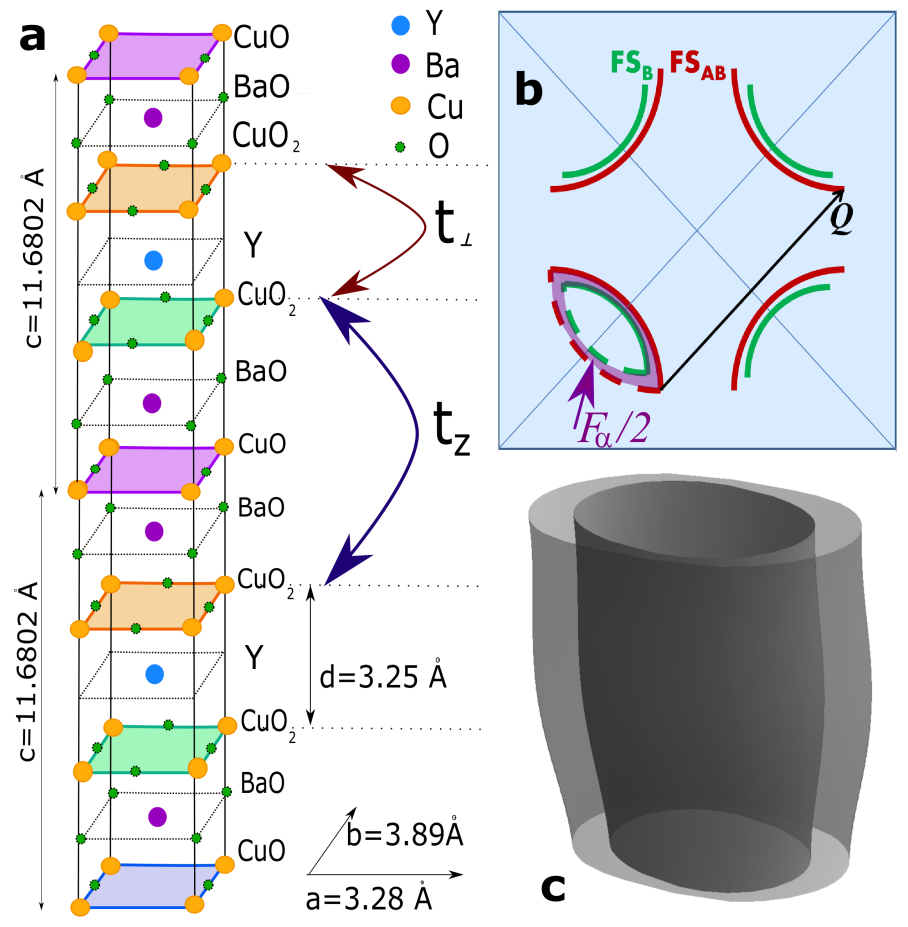

FIG. 1: The bilayer crystal structure and Fermi surface of YBCO. a) The crystal structure in YBCO, producing the bilayer splitting to bonding (B) and antibonding (AB) electron states and Fermi-surface parts. b) The schematically shown in-plane Fermi surface(FS) in YBCO, seen by ARPES and without fine-grained reconstruction. The solid green lines show Fermi arcs of bonding FS, and the red lines show the antibonding FS. The dashed lines show the FS parts shifted by the vector $Q=(\pi / a, \pi / b)$. The dashed and solid lines together form two closed FS pockets, corresponding to bonding and antibonding states and responsible for the $F_{\beta} \sim 1.6 k T$ frequency, about $6 \%$ of the Brillouin zone. The most prominent $F_{\alpha}$ frequency $\sim 2 \%$ of Brillouin zone corresponds to double the difference between green and red FS pockets, i.e. between $\mathrm{B}$ and AB FS pockets - the area shaded in purple. c) The illustration of a quasi-2D Fermi surface with interlayer warping due to $2 t_{z}$ and double bilayer splitting due to $2 t_{\perp}$.

edly much stronger, and magnetic oscillations from closed pockets should be even more strongly damped compared to the beat frequencies.

To pursue this basic idea, we here extend the theory developed for the organics to include interplanar couplings from the underlying bilayer structure of YBCO, illustrated in Fig. 1 The richer structure that emerges will, in fact, give a much clearer indication of the origin of the low-frequency oscillations. In a bilayer structure there are two types of interlayer hopping: (i) between adjacent layers separated by distance $d$ within one bilayer, given by the transfer integral $t_{\perp}=t_{\perp}\left(\boldsymbol{k}_{\|}\right)$, and (ii) between adjacent equivalent bilayers separated by distance $h$, given by the transfer integral $t_{z}=t_{z}\left(\boldsymbol{k}_{\|}\right)$, where $\boldsymbol{k}_{\|}$is the intralayer momentum. The resulting electron energy 
spectrum is given by $\stackrel{42}{\underline{2}}$

$\epsilon_{ \pm}\left(k_{z}, \boldsymbol{k}_{\|}\right)=\epsilon_{\|}\left(\boldsymbol{k}_{\|}\right) \pm \sqrt{t_{z}^{2}+t_{\perp}^{2}+2 t_{z} t_{\perp} \cos \left[k_{z}(h+d)\right]}$.

This electron energy spectrum has, for $t_{z} \ll t_{\perp}$, bonding and anti-bonding states each with weak $k_{z}$ dispersion and separated by $\sim 2 t_{\perp}\left(\boldsymbol{k}_{\|}\right)$:

$\epsilon_{ \pm}\left(k_{z}, \boldsymbol{k}_{\|}\right) \approx \epsilon_{\|}\left(\boldsymbol{k}_{\|}\right) \pm t_{\perp}\left(\boldsymbol{k}_{\|}\right) \pm 2 t_{z}\left(\boldsymbol{k}_{\|}\right) \cos \left[k_{z}(h+d)\right]$.

The corresponding in-plane Fermi surface, shown schematically in Fig. 10, contains four splitted Fermi arcs shown by green lines for bonding and by red lines for antibonding states, in agreement with ARPES data28,29. The dashed lines in Fig. 1 $1 b$ denote the Fermi arcs shifted by the vector $Q \approx(\pi / a, \pi / b)$, which correspond to the reconstructed Fermi surface due to scattering by AFM or pseudogap ordering. These dashed lines together with solid lines form two closed Fermi-surface pockets of slightly different area, which may produce MQO. According to our proposal, the doubled difference between the bonding (green) and antibonding (red) pockets gives the slow $F_{\alpha} \approx 530 T$ MQO frequency observed in YBCO. The two side frequencies come from $3 \mathrm{D}$ warping of these FS pockets, illustrated in Fig. 11 and originating from interbilayer electron hopping $t_{z}$, corresponding to the last term in Eq. (2). This interpretation is supported by the observed angular dependence of the $\Delta F_{\alpha}$ splitting ${ }^{24}$. Below we give a more quantitative and detailed substantiation of our interpretation.

\section{B. Analytical formula for slow magnetoresistance oscillations}

According to Eq. (2), in YBCO there should be at least two types of splitting of the original frequencies: the larger bilayer splitting $\Delta F_{\perp}=t_{\perp} B / \hbar \omega_{c}$, where $t_{\perp}=$ $\left\langle t_{\perp}\left(\boldsymbol{k}_{\|}\right)\right\rangle \neq 0$ and the angular brackets signify an averaging over in-plane momentum $\boldsymbol{k}_{\|}$on the Fermi surface, and the smaller splitting $\Delta F_{c}=2 t_{z} B / \hbar \omega_{c} \ll \Delta F_{\perp} \ll F_{\beta}$ due to the $k_{z}$ electron dispersion, where we also assume $t_{z}=\left\langle t_{z}\left(\boldsymbol{k}_{\|}\right)\right\rangle \neq 0$. These two splittings result in four underlying frequencies $F_{\beta} \pm \Delta F_{\perp} \pm \Delta F_{c}$ of similar amplitudes. The slow oscillations in magnetoresistance originate from these four frequencies result in a much richer set of frequencies than for a single layer structures previously considered, which had only two $F_{\beta} \pm \Delta F_{c} \underline{\underline{51,53}}$

The metallic conductivity along $i$-th axis $\sigma_{i}=\sigma_{i i}$ is given by the sum of contributions from all ungapped pockets $\beta$ and over two spin components $s$ :

$$
\sigma_{i}=\sum_{\beta, s} \sigma_{i, \beta, s}=\sum_{\beta, s} e^{2} g_{F \beta} D_{i, \beta} .
$$

At low temperature each pocket $\beta$ contributes to the total metallic conductivity along axis $i$ at low temperature via the product of a density of electron states (DoS)
$g_{F, \beta}=g_{\beta}\left(\varepsilon=E_{F}\right)$ and an electron diffusion coefficient $D_{i, \beta}$. Both contribute to oscillations, since they vary with the magnetic field $B_{z}$ perpendicular to the conducting $x-y$ layers as ${ }^{55}$ (see Appendix A for a detailed derivation)

$$
\frac{g_{F \beta}}{g_{0 \beta}}=1-\sum_{l= \pm 1} 2 J_{0}\left(2 \pi \frac{\Delta F_{c}}{B_{z}}\right) \cos \left(2 \pi \frac{F_{\beta}-l \Delta F_{\perp}}{B_{z}}\right) R_{D},
$$

where $R_{D}=\exp \left(-2 \pi^{2} T_{D} / \hbar \omega_{c}\right)$ is the Dingle factor ${ }^{54}$, and

$$
\frac{D_{i, \beta}}{D_{0 i, \beta}}=1+B_{i, \beta} \sum_{l= \pm 1} J_{0}\left(2 \pi \frac{\Delta F_{c}}{B_{z}}\right) \cos \left(2 \pi \frac{F_{\beta}-l \Delta F_{\perp}}{B_{z}}\right) R_{D} .
$$

Substituting Eqs. (47),(5) into Eq. (3) one obtains four types of oscillating terms: (i) the first harmonics, of first order in $R_{D}$ and oscillating rapidly at frequencies $\sim F_{\beta}$; (ii) the second harmonics with amplitude $\sim R_{D}^{2}$ and frequency $\sim 2 F_{\beta}$; (iii) "Ultra-slow" oscillations

$$
\sigma_{U S l O}\left(B_{z}\right) \propto J_{0}^{2}\left(2 \pi \Delta F_{c} / B_{z}\right) R_{D}^{2}
$$

with frequency $\sim 2 \Delta F_{c}$; and (iv) "Slow" oscillations with frequency $\sim 2 \Delta F_{\perp}$ :

$$
\sigma_{S l O}\left(B_{z}\right) \propto J_{0}^{2}\left(2 \pi \frac{\Delta F_{c}}{B_{z}}\right) \cos \left(4 \pi \frac{\Delta F_{\perp}}{B_{z}}\right) R_{D}^{2} .
$$

The Fourier transform (FT) of this magnetic-field dependence of $\sigma_{S l O}\left(B_{z}\right)$ is shown in Fig. 2. It closely resembles the experimental data in YBCO,$\frac{5,6,24}{2}$. There are three equidistant harmonics, and the amplitudes of the side peaks relative to the central decrease both with the Dingle factor $R_{D}$ and with a reduction of the field interval available in experiment.

\section{Temperature dependence of slow magnetoresistance oscillations}

At finite temperature $T$ the conductivity tensor is

$$
\sigma(T)=\int d \varepsilon\left[-n_{F}^{\prime}(\varepsilon)\right] \sigma(\varepsilon)
$$

where the derivative of the Fermi distribution function $n_{F}^{\prime}(\varepsilon)=-1 /\left\{4 T \cosh ^{2}[(\varepsilon-\mu) / 2 T]\right\}$, and $\sigma_{i j}(\varepsilon)$ is the component of the electron conductivity tensor at energy $\varepsilon$. Usual magnetic oscillation come from the first-order terms in the Dingle factor, such as $g_{F \beta}(\varepsilon) / g_{0 \beta}$, which oscillate rapidly as a function of energy $\varepsilon$ :

$$
\frac{\sigma_{1}(\varepsilon)}{\sigma_{0}} \propto 2 J_{0}\left(\frac{4 \pi t_{z}}{\hbar \omega_{c}}\right) R_{D} \sum_{ \pm} \cos \left(2 \pi \frac{\varepsilon \pm t_{\perp}}{\hbar \omega_{c}}\right)
$$

where $\sigma_{0}$ is the non-oscillating part of conductivity. Substituting Eq. (9) into Eq. (8) and performing the integration over $\varepsilon$, one obtains the well-known result that at finite temperature the MQO are damped by the factor

$$
R_{T}=\left(2 \pi^{2} k_{B} T / \hbar \omega_{c}\right) / \sinh \left(2 \pi^{2} k_{B} T / \hbar \omega_{c}\right),
$$



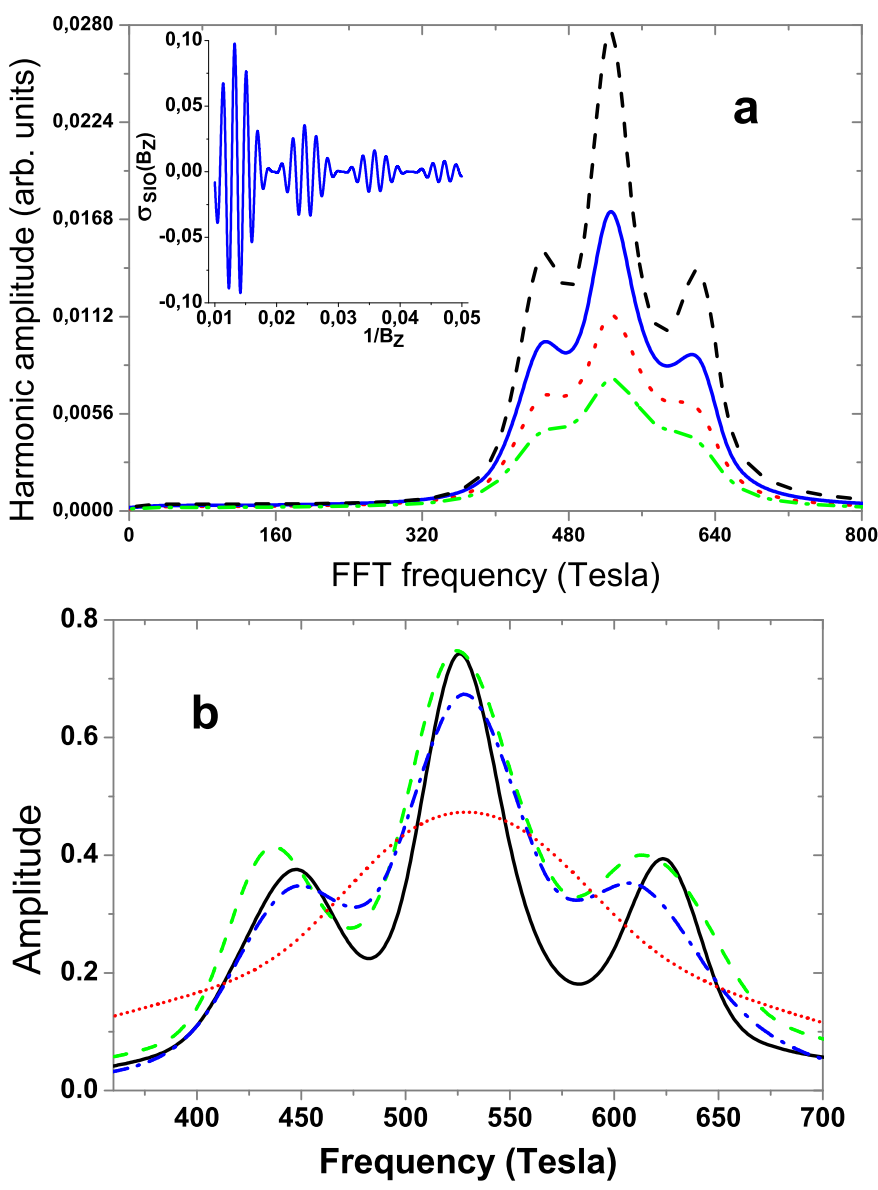

FIG. 2: Predicted magnetic oscillations in quasi-2D conductor with bilayer splitting. (a) The Fourier transform of magnetic oscillations given by Eq. (17) at four different Dingle temperatures $\pi T_{D} / \hbar \omega_{c}\left(B_{z}=1 T\right)=1$ (dashed black line), 3 (solid blue line), 5 (dotted red line) and 7 (dash-dotted green line). Insert shows the initial function $\sigma_{S l O}\left(1 / B_{z}\right)$ at $\pi T_{D} / \hbar \omega_{c}\left(B_{z}=1 T\right)=3$. (b) The Fourier transform of conductivity in Eq. (7) at $T_{D}=0$ but in the finite field intervals $20 T<B_{z}<100 T$ (solid black line), $20 T<B_{z}<65 T$ (dashed green line), $25 T<B_{z}<65 T$ (dash-dotted blue line), and $30 T<B_{z}<65 T$ (dotted red line).

which gives

$$
\begin{aligned}
& \frac{\sigma_{1}(\mu)}{\sigma_{0}} \propto 2 J_{0}\left(\frac{4 \pi t_{z}}{\hbar \omega_{c}}\right) \cos \left(2 \pi \frac{\mu \pm t_{\perp}}{\hbar \omega_{c}}\right) R_{D} R_{T} \\
= & 2 J_{0}\left(\frac{2 \pi \Delta F_{c}}{B_{z}}\right) \sum_{l= \pm 1} \cos \left(2 \pi \frac{F_{\beta}-l \Delta F_{\perp}}{B_{z}}\right) R_{D} R_{T} .
\end{aligned}
$$

The slow oscillations come from the product of oscillating quantities, such as $\left(g_{F \beta}(\varepsilon) / g_{0 \beta}\right)^{2}$, which for bilayersplitted electron dispersion in Eq. (2) in the second order in $R_{D}$ gives

$$
\begin{gathered}
\sigma_{2}(\varepsilon) \propto 4 J_{0}^{2}\left(\frac{4 \pi t_{z}}{\hbar \omega_{c}}\right) \cos \left(2 \pi \frac{\varepsilon-t_{\perp}}{\hbar \omega_{c}}\right) \cos \left(2 \pi \frac{\varepsilon+t_{\perp}}{\hbar \omega_{c}}\right) R_{D}^{2} \\
=2 J_{0}^{2}\left(\frac{4 \pi t_{z}}{\hbar \omega_{c}}\right)\left[\cos \left(\frac{4 \pi \varepsilon}{\hbar \omega_{c}}\right)+\cos \left(\frac{4 \pi t_{\perp}}{\hbar \omega_{c}}\right)\right] R_{D}^{2} .
\end{gathered}
$$

The first term in the square brackets gives the second harmonic of MQO, which is strongly damped by temperature by the factor close to the square of $R_{T}$ in Eq. (10) similarly to the first harmonic. The second term in the square brackets of Eq. (12), responsible for $\mathrm{SlO}$, is independent of energy. Therefore, its integration over $\varepsilon$ with $n_{F}^{\prime}(\varepsilon)$ in Eq. (8) does not produce any temperature damping factor for $\mathrm{SlO}$ :

$\sigma_{2}(\mu) \propto J_{0}^{2}\left(\frac{4 \pi t_{z}}{\hbar \omega_{c}}\right)\left[\cos \left(\frac{4 \pi \mu}{\hbar \omega_{c}}\right) R_{T}^{2}+\cos \left(\frac{4 \pi t_{\perp}}{\hbar \omega_{c}}\right)\right] R_{D}^{2}$.

Thus, within this simplest model, the $\mathrm{SlO}$ are not damped by temperature, as seen in the observed slow oscillations in Refs $: \stackrel{51,52}{~}$. However, magnetoresistance oscillations observed at frequency $F_{\alpha} \approx 530 T$ have some temperature damping, corresponding to an effective mass of $m^{\star} \approx 1.6 m_{e} \frac{22,24,63}{2}$ Such a strong temperature damping of what are proposed by us as being slow oscillations may arise from the square of the temperature-dependent Dingle factor $R_{D}, \underline{64}$ For non-interacting electrons, when the Dingle factor comes only from impurity scattering, $R_{D}$ is almost temperature independent. A weak electronphonon interaction was also predicted ${ }^{65}$ not to violate the usual $R_{T}(T)$ to the lowest-order perturbation theory, but for YBCO at $T \sim 10 \mathrm{~K}$ the electron-phonon interaction is not weak, and the result of Ref $\frac{65}{6}$ may not apply. In addition, the electron-electron interaction, which in cuprates is very strong, gives considerable temperature dependence to $R_{D} \stackrel{66}{=}$ as observed experimentally and may contribute to the measured effective electron mass $m^{\star} \approx 1.6 m_{e}$. Note that the extracted effective mass depends strongly on doping outside the doping interval $0.1<p<0.125, \frac{22}{2}$ and this would reflect the doping dependence of the strength of e-e interactions.

\section{Effect of macroscopic spatial inhomogeneities}

The macroscopic spatial inhomogeneities affect MQO similarly to temperature, because they smear the Fermi level $\mu$ along the whole sample. To show explicitely, let us take the most common Gaussian distribution of the spatially fluctuating shift of Fermi level $\Delta \mu(r)$, given by the normalized weight

$$
D(\Delta \mu)=(1 / \sqrt{2 \pi} W) \exp \left[-(\Delta \mu)^{2} / 2 W^{2}\right] .
$$

In addition to the temperature smearing in Eq. (8), given by the integration over electron energy $\varepsilon$, conductivity acquires the coordinate smearing, given by the integration over the shift $\Delta \mu(r)$ of chemical potential weighted by Eq. (14):

$$
\sigma=\int d \mu \sigma(\mu) D\left(\mu-\mu_{0}-\Delta \mu\right),
$$

where $\sigma(\mu)$ is given by the sum of first- and second-order terms given by Eqs. (11) and (13). The first-order terms 
give the MQO

$$
\begin{gathered}
\frac{\sigma_{1}}{\sigma_{0}} \propto \int d \mu D\left(\mu-\mu_{0}-\Delta \mu\right) 2 J_{0}\left(\frac{4 \pi t_{z}}{\hbar \omega_{c}}\right) \\
\times \cos \left(2 \pi \frac{\mu \pm t_{\perp}}{\hbar \omega_{c}}\right) R_{D} R_{T} \\
=2 J_{0}\left(\frac{4 \pi t_{z}}{\hbar \omega_{c}}\right) \cos \left(2 \pi \frac{\mu_{0} \pm t_{\perp}}{\hbar \omega_{c}}\right) R_{D} R_{T} R_{W} .
\end{gathered}
$$

Here the last damping factor

$$
R_{W}=\exp \left(-2 \pi^{2} W^{2} / \hbar^{2} \omega_{c}^{2}\right)
$$

comes from the spatial variations of the Fermi level. The width $W$ of this Gaussian distribution of Fermi level contributes to the total Dingle temperature $T_{D}^{\text {tot }} \approx$ $T_{D}^{i m p}+T_{D}^{i n h}$, where $T_{D}^{i n h} \approx W$. Note that the Gaussian Fermi-level smearing leads to the quadratic dependence of $\ln R_{W}$ on magnetic field and on harmonic number, which was experimentally observed in high-quality samples of quasi-2D organic metals $\underline{56}$

The second-order terms (13) after substitution to Eq. (15) give

$$
\begin{gathered}
\frac{\sigma_{2}}{\sigma_{0}} \propto \int d \mu D\left(\mu-\mu_{0}-\Delta \mu\right) J_{0}^{2}\left(\frac{4 \pi t_{z}}{\hbar \omega_{c}}\right) R_{D}^{2} \\
\times\left[\cos \left(\frac{4 \pi \mu}{\hbar \omega_{c}}\right) R_{T}^{2}+\cos \left(\frac{4 \pi t_{\perp}}{\hbar \omega_{c}}\right)\right] \\
=\left[\cos \left(\frac{4 \pi \mu_{0}}{\hbar \omega_{c}}\right) R_{T}^{2} R_{W}^{4}+\cos \left(\frac{4 \pi t_{\perp}}{\hbar \omega_{c}}\right)\right] J_{0}^{2}\left(\frac{4 \pi t_{z}}{\hbar \omega_{c}}\right) R_{D}^{2} .
\end{gathered}
$$

We see, that the second harmonic acquires the enhanced damping factor $R_{W}^{4}$ from the Fermi-level variations, while the slow oscillations remain unchanged.

As a result, the fast quantum oscillations are additionally damped by the Fermi-level variations, while the $\mathrm{SlO}$ are not affected by this type of disorder $\underline{51,52}$ Qualitatively, this can be described by a complex Dingle factor $R_{D}^{\text {tot }}=R_{D} R_{W}$, or as a complex Dingle temperature $T_{D}^{\text {tot }} \approx T_{D}+W \underline{\underline{51}}$ The first usual part of the Dingle factor $R_{D}$ comes from short-range disorder, e.g. impurities, and enters both the usual fast MQO and SlO. The second part of the Dingle factor $R_{W}$ comes from the smearing of the local Fermi level by macroscopic longrange inhomogeneities and enters only the fast MQO. In organic metals the second part of the Dingle temperature $W$ turns out to be unexpectedly large, being more than four times larger than the first part $T_{D}$, so that $T_{D}^{t o t} \approx 5.3 T_{D}$, as was demonstrated from the experimental data on the field dependence of the amplitudes of both types of magnetic oscillations in $\beta$-(BEDT-TTF) ${ }_{2} \mathrm{IBr}_{2}, \underline{{ }^{51}}$ In cuprates, which are notoriously inhomogeneous, the difference between $T_{D}^{\text {tot }}$ and $T_{D}$ can be even larger. Using Eqs. (16) and (17) and neglecting the second harmonics $\propto \cos \left(4 \pi \mu_{0} / \hbar \omega_{c}\right)$ in Eq. (17), we can rewrite the conductivity $\sigma=\sigma_{1}(\mu)+\sigma_{2}(\mu)$ as

$$
\begin{aligned}
\frac{\sigma}{\sigma_{0}}= & J_{0}\left(\frac{4 \pi t_{z}}{\hbar \omega_{c}}\right) \sum_{l= \pm 1} \cos \left(2 \pi \frac{\mu+2 l t_{\perp}}{\hbar \omega_{c}}\right) R_{T} R_{D} R_{W} \\
& +J_{0}^{2}\left(\frac{4 \pi t_{z}}{\hbar \omega_{c}}\right) \cos \left(\frac{4 \pi t_{\perp}}{\hbar \omega_{c}}\right) R_{D}^{2} .
\end{aligned}
$$

The first line describes usual MQO, probably $F_{\beta}$ frequency in $\mathrm{YBCO}$, while the second line describes $\mathrm{SlO}$, i.e. $F_{\alpha}$ and $F_{\alpha} \pm \Delta F$ frequencies in YBCO. The damping factor $R_{T} R_{D} R_{W}$ of usual MQO is, probably, much sronger than the damping factor $R_{D}^{2}$ of $\mathrm{SlO}$, which explains why $F_{\beta}$ frequency in YBCO is much weaker and more fragile than $F_{\alpha}$ and even than $F_{\alpha} \pm \Delta F$ frequencies.

\section{E. Angular dependence of the frequencies of slow magnetoresistance oscillations}

The angular dependence of the split frequency $2 \Delta F_{c}$, proportional to $t_{z}$, drastically differs from that of $2 \Delta F_{\perp}$, related to the bilayer splitting $t_{\perp}$. The frequency $2 \Delta F_{c} \propto$ $t_{z}$ has a strongly non-monotonic dependence on the tilt angle $\theta$ of the magnetic field: $\underline{51,57}$

$$
\Delta F_{c}(\theta)=\Delta F_{c}(\theta=0) J_{0}\left(k_{F} c^{\star} \tan \theta\right) / \cos \theta,
$$

where $c^{\star}(=11.65 \AA$ for $\mathrm{YBCO})$ is the lattice constant in the interlayer $z$-direction, and $k_{F}$ is the Fermi momentum. This angular dependence is the same as for the beat frequency of MQO in a quasi-2D meta ${ }^{51}$, but differs strongly from the standard cosine dependence

$$
F(\theta)=F(\theta=0) / \cos \theta,
$$

typical for quasi-2D metals where the interplanar coupling is so small that the Fermi surface can be considered perfectly cylindrical. Eq. (19) has an obvious geometrical interpretation ${ }^{58}$ : the slightly warped Fermi surface has two extremal cross-sections $S_{\text {ext }}$ perpendicular to the magnetic field $\boldsymbol{B}$, which become equal, to first order in $t_{z}$, at some tilt angles $\theta_{Y a m}$. In contrast $2 F_{\perp}$ has the cosine angular dependence given by Eq. (20). To see this, consider the dispersion in Eq. (2) at $t_{z} \ll t_{\perp}$, corresponding to the Fermi surface in Fig. 15. The Fermi surface consists of two cylinders along the $z$-axis with base areas $S_{\text {even }}$ and $S_{\text {odd }}$, differing by $\Delta S=S_{\text {even }}-S_{\text {odd }}=4 \pi t_{\perp} m^{*}=$ const. In a tilted magnetic field the two corresponding extremal cross-section areas are $S_{\text {even,odd }}=S_{\text {even,odd }} / \cos \theta$, leading directly to Eq. (20). Thus, according to our interpretation, the angular dependence of $F_{\alpha}$ frequency from bilayer splitting obeys the usual cosine dependence given by Eq. (20), while the shift $\Delta F_{\alpha}$ of shoulder frequencies obeys Eq. (19) in agreement with experimental data in Ref $\frac{24}{2}$. 


\section{DISCUSSION}

In the previous section we have shown that the interlayer hopping may produce low-frequency magnetic oscillations, given by Eq. (17) and illustrated in Fig. 2] Although these slow oscillations contain usual Dingle factor squared, they may be much stronger than the usual MQO, because the latter are strongly damped by sample inhomogeneities (see Sec. IID) or by temperature (see Sec. IIC). The Fourier transform of these slow oscillations has a natural three-peak structure. The amplitude of the central frequency $2 \Delta F_{\perp}$ is at least twice as large as the amplitudes of the side frequencies $2 \Delta F_{\perp} \pm 2 \Delta F_{c}$, as can be seen from pure combinatorics ${ }^{59}$ (see Appendix $\mathrm{B}$ for details). As given by Eq. (7) and shown in Fig. 2. the amplitudes of side peaks are additionally damped by the Dingle factor or due to the finite field range of available experimental data. This theoretical plot closely resembles the experimental data in $\mathrm{YBCO}, \underline{-7}, 24$. We therefore propose that the observed ${ }^{4,20-27}$ three equidistant harmonics of magnetic oscillations at low frequency $F_{\alpha} \approx 530 T$ in $\mathrm{YBa}_{2} \mathrm{Cu}_{3} \mathrm{O}_{6+\delta}$ (and, probably, in $\mathrm{YBa}_{2} \mathrm{Cu}_{4} \mathrm{O}_{8} \stackrel{46}{-48}$ ) are not due to the tiny pockets of the Fermi surface reconstructed by CDW order $,-7,41-45$ but originate from mixing of four frequencies $F_{\beta} \pm \Delta F_{\perp} \pm \Delta F_{c}$, formed by a fundamental frequency $F_{\beta}$ split by bilayer and interbilayer electron hopping integrals $t_{\perp}$ and $t_{z}$. Several terms come from this frequency mixing even in the lowest second order in Dingle factor. A half of these terms come from various sums of fundamental frequencies and correspond to the second harmonics of fundamental frequencies, being much weaker than MQO with fundamental frequencies appearing in the first order in Dingle factor. The second half of mixed frequencies correspond to the various differences of fundamental frequencies, which do not contain electron energy or Fermi level but only split energies. Hence, these oscillations are robust against sample inhomogeneities, and therefore are much more pronounced than the MQO with fundamental frequencies. Such oscillations are responsible for the observed three-peak Fourier transform. The corresponding analytical expression is given in Eq. (7). The frequencies $F_{\alpha}=2 \Delta F_{\perp} \approx 530 T=2 t_{\perp} B / \hbar \omega_{c}$ and $\Delta F_{\alpha}=2 \Delta F_{c} \approx 90 T \approx 4 t_{z} B / \hbar \omega_{c}$ provide an experimental measure of the values of bilayer $t_{\perp}$ and inter-bilayer $t_{z}$ average electron transfer integrals. The extracted bilayer splitting can also be compared to ARPES data 29 . We summarize arguments for this new interpretation:

(1) Frequency pattern. The multiple extra magnetic oscillation frequencies predicted from the scenarious with Fermi surface reconstruction are missing in experiment. ${ }^{-8,41-45}$. In contrast, the proposed model predicts only the three peaks shown in Fig. 2, The only frequency with an amplitude comparable to the side peaks at $F_{\alpha} \pm \Delta F_{\alpha}$ would be even lower: $2 \Delta F_{c} \sim 90 T$. The corresponding analytical expression is given in Eq. (6). Such ultraslow oscillations could be detected only at low magnetic field $B_{z}<2 \Delta F_{c}$, where they are strongly damped by the Dingle factor. In addition, these ultraslow oscillations are not exactly periodic in $1 / B_{z}$, especially in high field $B_{z} \sim \Delta F_{c}$, because the zeros of the Bessel's function in Eq. (6) are not exactly equidistant. Nevertheless, such low-frequency magnetic oscillations have been observed recently ${ }^{27}$ supporting the proposed interpretation. Our model also predicts a much larger frequency $F_{\beta}$ from true Fermi surface pockets but with much smaller amplitude and much stronger damped by temperature and spatial inhomogeneities. Experimentally, the $F_{\beta} \approx 1.65 \mathrm{kT}$ frequency was indeed observed in $\mathrm{dHvA}^{20}$ and Tunnel Diode Oscillation cyclotron resonance 23 measurements, but only at very low temperatures and not in all samples 8 .

(2) Agreement with ARPES. The value of the bilayer splitting $\Delta_{B S}=0.075 \AA^{-1}$ in momentum space observed by $\mathrm{ARPES}^{29}$ is consistent with the $F_{\alpha}=2 \Delta F_{\perp}=$ $530 T$ frequency, corresponding to the FS area $S_{\alpha}=$ $0.0507 \AA^{-1} \approx 2 \% \cdot S_{B Z}$. According to our interpretation it is the doubled shaded area between the bonding and antibonding Fermi arcs (see Fig. 1b), given by the product $4 \Delta_{B S} l_{F A}$, where $l_{F A} \approx 0.17 \AA^{-1}$ is the length of a Fermi arc. The value of the average bilayer splitting $t_{\perp}$ expected from band-structure calculations 45,60 is also consistent with $F_{\alpha}: 62 t_{\perp} \equiv\left\langle t_{\perp}\left(\boldsymbol{k}_{\|}\right)\right\rangle=\hbar e F_{\alpha} / 2 m_{\beta}^{*} \approx$ $8 \mathrm{meV}$. Note that the maximum value $t_{\perp}\left(\boldsymbol{k}_{\|}\right)$of bilayer transfer integral may considerably exceed this average value $t_{\perp}$. Similarly the observed $t_{z}$-induced splitting $2 \Delta F_{c} \approx 90 T \approx 4 t_{z} B / \hbar \omega_{c}$ gives a reasonable average value $2 t_{z} \approx 1.4 \mathrm{meV}$.

(3) Doping dependence. The observed $F_{\alpha} \approx 530 T$ depends weakly on the degree of doping $22,23,50$, more consistent with our model than expected ${ }^{49}$ from tiny pockets in the Fermi-surface appearing due to FS reconstruction.

(4) Damping by sample inhomogeneities. Long-range spatial inhomogeneities, common in cuprates, should strongly damp oscillations from true Fermi-surface pockets due to the smearing of Fermi level similar to the effect of temperature (see Secs. IIC and IID). This type of disorder affects the proposed slow oscillations much less, as shown in Sec. IID, making their observation much easier.

(5) The angular dependencies of the observed frequencies $^{24} F_{\alpha}$ and $\Delta F_{\alpha}$ agree with Eqs. (20) and (19) correspondingly. The observed strong angular dependence of the split frequency $\Delta F_{c}(\theta)$ (see Fig. 4a in Ref. $\frac{24}{}$ ) is well fit by Eq. (19), suggesting that this frequency is indeed related to the $k_{z}$ electron dispersion, coming from the interlayer transfer integral $t_{z}$ and giving the warping of a larger Fermi surface. The extracted ${ }^{24}$ first Yamaji angle $\theta_{Y a m} \approx 43^{\circ}$ in $\Delta F_{c}(\theta)$, corresponding to the first zero of the Bessel function $J_{0}\left(k_{F} c^{\star} \tan \theta\right)$, indicates the Fermi momentum $k_{F}=2.4 / c^{\star} \tan \theta_{Y a m}=2.2 \mathrm{~nm}^{-1}$ and a true Fermi-surface area of about $S_{\text {ext }} \sim \pi k_{F}^{2} \approx$ $15 \mathrm{~nm}^{-2}$. This corresponds to an underlying quantum oscillation frequency of $S_{\text {ext }} \hbar / 2 \pi e \approx 1.6 \mathrm{kT}$, which is far from $F_{\alpha} \approx 530 \mathrm{~T}$ but close to the reported value of $F_{\beta} \approx 1.65 \mathrm{kT}^{20,23}$. Note that $F_{\beta}$ was observed only at rather low temperatures and not in all samples,,$\frac{8}{,}$ which 
is consistent with our model, because frequencies corresponding to real Fermi-surface pockets are stronger damped by sample-dependent inhomogeneities and by temperature than slow oscillations.

(6) Insensitivity to magnetic breakdown. A CDWinduced Fermi-surface reconstruction is not necessary for the observation of SlO. Hence, there are no issues to be resolved as how a weak and fluctuating CDW ordering, with transition temperature less than $40 \mathrm{~K}$, overcomes the magnetic breakdown, which should be strong for fields up to 100 tesla and restore the electron orbits of unreconstructed Fermi surface.

(7) The relative amplitudes of the observed frequencies are naturally explained without additional fitting parameters (see Fig. 2).

Very similar magnetic oscillations have been observed in another closely related bilayer stoichiometric high-Tc superconductor $\mathrm{YBa}_{2} \mathrm{Cu}_{4} \mathrm{O}_{8} \cdot \underline{46}-\underline{48}$ This compound has no indication of a CDW order, which excludes the CDWinduced fine-grained FS reconstruction giving small FS pockets. Hence, the proposed magnetic oscillations due to bilayer splitting is a quite general phenomenon. Not only cuprates but also rare-earth tritelluride compounds with bilayer crystal structure show similar slow oscillations 53 originating from bilayer splitting, but the three-peak structure is not resolved there because of a very small value of $t_{z}$. Similar slow oscillations with a frequency $\approx 840 T$ (but without side-frequency splitting), corresponding to $3 \%$ of the Brillouin zone, have been observed in $\mathrm{HgBa}_{2} \mathrm{CuO}_{4+\delta}$, where there is no bilayer splitting, but a much larger $t_{z}$ is expected from the shorter interlayer distance. It is as yet unclear whether this frequency has the same origin, coming from $t_{z}$, spin-orbit, magnetic or some other type of splitting. Therefore, a more detailed study of these magnetic oscillations, as well as the further theoretical study of this effect taking into account various types of electron-band splitting is need.

To summarize, we propose and analyze a new interpretation of the observed magnetic oscillations in $\mathrm{YBa}_{2} \mathrm{Cu}_{3} \mathrm{O}_{6+\delta}$ high-Tc superconductors, with frequencies $F_{\alpha} \approx 530 T$ and $F_{\alpha} \pm \Delta F_{\alpha}$ related to the bilayer splitting and $t_{z}$-dispersion rather than to tiny Fermi-surface pockets. This is based on the new result (7), as illustrated in Fig. 2. The frequencies agree with ARPES data, thus resolving the long-standing puzzle, and allow us to estimate values of bilayer splitting $t_{\perp}=\hbar e F_{\alpha} / 2 m^{*}$ and of $k_{z}$-dispersion $t_{z}=\hbar e \Delta F_{\alpha} / 4 m^{*}$. The observed angular dependence of $\Delta F_{\alpha}$ points to a true Fermi-surface pocket close to $F_{\beta} \approx 1.6 \mathrm{kT}$. This interpretation is robust, requiring only a bilayer crystal structure and closed Fermi-surface pockets. The reproducibility of $F_{\alpha} \approx 530 T$ and $F_{\alpha} \pm \Delta F_{\alpha}$ frequencies in $\mathrm{YBCO}$, in contrast to that of the more fragile $F_{\beta} \approx 1.6 \mathrm{kT}$, is understood in terms of the lesser sensitivity to sample inhomogeneities. The comparison of the amplitudes of $F_{\alpha}$ and $F_{\beta}$ oscillations then provides a potentially useful way of characterizing the type of disorder present in a given sample. Verifying the existence, and, in principle a further splitting of the
$F_{\beta}$, in extremely clean samples would be a good test of the proposed theory.

This brings us back to the issue of the charge density order, where we have mentioned there is now mounting evidence, particularly at the high fields where magnetic oscillations are observed $\stackrel{34}{\underline{3}}$ While we have argued that the magnetic oscillations that were seen should not be taken as evidence of any corresponding detailed Fermi surface reconstruction, we cannot rule out the possibility of such reconstruction. Any new oscillations would be subject to magnetic breakdown, which depends on the strength of charge density order and the disorder. It would be important to quantify both aspects fully, by detailed study of charge order and magnetic oscillation as a function of doping and applied pressure, but it is likely that any new oscillations would be more fragile than the oscillations we describe. In our view, the bilayer geometry of YBCO is ideal in identifying the source of the oscillations that have been seen, but it could well be that in single-layer cuprates a similar mechanism can explain low frequency components.

The proposed model not only resolves the puzzle of inconsistency between ARPES and MQO data, but also suggests how the available extensive experimental data on MQO in YBCO can be used. According to our interpretation, the frequency of the observed slow magnetic oscillations in YBCO gives the area between bonding and antibonding Fermi-surface arcs much more precisely than can be obtained from ARPES data ${ }^{29}$ The angular dependence of the observed shoulder frequencies allows to estimate the areas of the true Fermi-surface pockets, which may stimulate their reliable experimental observation. The comparison of the amplitudes of $\mathrm{SlO}$ and true MQO would give the important information about the spatial variations of the Fermi level due to sample inhomogeneities. Our model also predict the magnetic oscillations with ultra-low frequency $\sim 90$ tesla, which has an unusual angular dependence given by Eq. (19). Possibly such oscillations were detected in Ref. ${ }^{27}$, but the angular dependence of their frequency needs further experimental study.

\section{Acknowledgments}

The authors thank Ted Forgan for raising this issue and communication of unpublished results. The work is partially supported by the Ministry of Education and Science of the Russian Federation in the framework of Increase Competitiveness Program of MISiS and by RFBR. P.G. acknowledges the RSCF grant \# 16-42-01100.

\section{Appendix A: Density of states in quasi-2D metal with bilayer crystal structure}

In a single-band quasi-2D metal without bilayer splitting with only one cylindrical Fermi Surface, an electron 
dispersion in the tight-binding approximation is 1

$$
\epsilon\left(k_{z}, \boldsymbol{k}_{\|}\right) \approx \epsilon_{\|}\left(\boldsymbol{k}_{\|}\right)-2 t_{z} \cos \left(k_{z} c^{\star}\right) .
$$

In magnetic field $\boldsymbol{B}$ the electron spectrum consists of a set of equidistant Landau levels separated by $\hbar \omega_{c}=$ $\hbar e B_{z} / m^{*} c$, where $m^{*}$ is the effective electron mass:

$$
\epsilon_{s}\left(k_{z}, n\right) \approx \hbar \omega_{c}(n+1 / 2)-2 t_{z} \cos \left(k_{z} c^{\star}\right)+s \Delta_{Z},
$$

where $\Delta_{Z}=\mathrm{g} \mu_{B} B$ is the Zeeman splitting, $\mathrm{g}$ is the $\mathrm{g}$ factor, $\mu_{B}$ is the Borh magneton, and $s= \pm 1 / 2$ is the projection of quasiparticle spin on magnetic field $\boldsymbol{B}$, inclined by the angle $\theta$ with respect to the normal to conducting layers. The corresponding oscillating density of electron states (DoS) $g(\varepsilon)$ is given by the sum over Landau levels (LL):

$$
\begin{gathered}
g(\varepsilon)=\sum_{n=0}^{\infty} \sum_{k_{z}, s} \delta\left[\varepsilon-\epsilon_{s}\left(k_{z}, n\right)\right] \\
=\sum_{n=0}^{\infty} \sum_{s= \pm 1} \frac{g_{L L} / \pi}{\sqrt{4 t_{z}^{2}-\left(\varepsilon-s \Delta_{Z}-\hbar \omega_{c}(n+1 / 2)+i \Gamma\right)^{2}}}
\end{gathered}
$$

where the LL degeneracy per unit area $g_{L L}=e B_{z} / 2 \pi \hbar c$, and $\Gamma=\Gamma(\varepsilon)$ is the broadening of electron levels due to scattering. It is often convenient to transform this sum over LL to the sum over harmonics using the Poisson summation formula. When MQO are weak, e.g. at $\Gamma \gtrsim \hbar \omega_{c}$, keeping only the zeroth and first harmonics one obtains: ${ }^{55}$

$$
\frac{g(\varepsilon)}{g_{0}}=1-2 J_{0}\left(\frac{4 \pi t_{z}}{\hbar \omega_{c}}\right) \cos \left(\frac{2 \pi \varepsilon}{\hbar \omega_{c}}\right) \cos \left(\frac{2 \pi \Delta_{Z}}{\hbar \omega_{c}}\right) R_{D},
$$

where $g_{0}=m^{*} / \pi \hbar^{2} d=2 g_{L L} / \hbar \omega_{c} d$ is the average DoS at the Fermi level per two spin components, $J_{0}(x)$ is the Bessel's function, and $R_{D}=\exp \left(-2 \pi \Gamma / \hbar \omega_{c}\right)$ is the Dingle factor $\stackrel{2}{2}$ The DoS at the Fermi level is found by making the subsititution $\varepsilon \rightarrow \mu$, where $\mu$ is the chemical potential equal to Fermi energy. Introducing the magnetic quantum oscillation frequency $F_{\beta}=\mu B_{z} / \hbar \omega_{c}$, we rewrite Eq. A2 as

$$
\frac{g_{F}}{g_{0}}=1-2 J_{0}\left(\frac{2 \pi \Delta F_{c}}{B_{z}}\right) \cos \left(2 \pi \frac{F_{\beta}}{B_{z}}\right) \cos \left(\frac{2 \pi m^{*}}{m \cos \theta}\right) R_{D} .
$$

For conductivity the sum over two spin components appears only in the final expression in Eq. (3), which contains the DoS per one spin component without factor $\cos \left(2 \pi m^{*} / m \cos \theta\right)$. Below we do not keep the Zeeman spin splitting $\Delta_{Z}$ and the spin subscript $s$ in the intermediate formulas, but similar to the standard calculations $\frac{1-3}{-3}$ introduce it only in the end as an energy shift by $\pm \Delta_{Z}$.

In bilayer crystals the quasi-2D electron dispersion (2) is additionally split by $2 t_{\perp}\left(\boldsymbol{k}_{\|}\right)$to the so-called "bonding" and "atibonding" states. The corresponding DoS for one spin component is given by the sum of contributions from the bonding and antibonding branches of electron dispersion

$$
g(\varepsilon)=\sum_{n=0}^{\infty} \sum_{ \pm} \frac{g_{L L} / \pi}{\sqrt{4 t_{z}^{2}-\left(\varepsilon \pm t_{\perp}-\hbar \omega_{c}(n+1 / 2)+i \Gamma\right)^{2}}}
$$

Applying the Poisson summation formula and keeping only the zeroth and first harmonics, similar to Eq. (A2), one obtains:

$$
\frac{g(\varepsilon)}{2 g_{0}}=1-\sum_{l= \pm 1} 2 J_{0}\left(\frac{4 \pi t_{z}}{\hbar \omega_{c}}\right) \cos \left(\frac{2 \pi \varepsilon-l t_{\perp}}{\hbar \omega_{c}}\right) R_{D},
$$

which gives the DoS on the Fermi level $\varepsilon=\mu$, corresponding to Eq. (4).

$$
\frac{g_{F}}{2 g_{0}}=1-\sum_{l= \pm 1} 2 J_{0}\left(\frac{2 \pi \Delta F_{c}}{B_{z}}\right) \cos \left(2 \pi \frac{F_{\beta}-l \Delta F_{\perp}}{B_{z}}\right) R_{D} .
$$

The diffusion coefficient $D_{i}$ depends on its direction $i$ with respect to magnetic field and to the crystal axes, and its oscillations can be approximated by

$\frac{D_{i}}{D_{0 i}} \approx 1-B_{i} J_{0}\left(\frac{2 \pi \Delta F_{c}}{B_{z}}\right) \sum_{l= \pm 1} \cos \left(2 \pi \frac{F_{\beta}-l \Delta F_{\perp}}{B_{z}}\right) R_{D}$,

where $D_{0 i}$ is the average non-oscillating part of diffusion coefficient and the coefficients $B_{i} \sim 1$ depend on the direction $i$. For $i \| z$ and without bilayer splitting it was calculated in Refs $\frac{51,52}{5}$, where in addition to $J_{0}\left(4 \pi t_{z} / \hbar \omega_{c}\right)$ the terms containing $J_{1}\left(4 \pi t_{z} / \hbar \omega_{c}\right)$ appear, which only introduce the phase shift of beats and of slow oscillations. For $i \perp z$ these $J_{1}\left(4 \pi t_{z} / \hbar \omega_{c}\right)$ terms are small by a factor $\sim t_{z} / E_{F}, \frac{61}{1}$ and Eq. (A7) is valid.

\section{Appendix B: Combinatorical explanation of the observed 3-peak structure of MQO}

The observed 3-peak structure of MQO Fourier transform, with the central peak at least twice larger than the side peaks, can be obtained from simple combinatorics without using the Bessel's functions. The combination of double bilayer splitting by $t_{\perp}\left(\boldsymbol{k}_{\|}\right)$and the Fermi-surface warping due to $t_{z}$ with two extremal FS cross-section areas gives a four-fold splitting of MQO frequency $F$ corresponding to the true FS pocket area both for the DoS $g_{F}$ and for the diffusion coefficient $D_{i}$ oscillate with varing of magnetic field $B_{z}$ perpendicular to the conducting $x-y$ layers as:

$$
\frac{g_{F}}{g_{0}}=1+A \sum_{j, l= \pm 1} \cos \left(2 \pi \frac{F_{\beta}+j \Delta F_{\perp}+l \Delta F_{c}}{B_{z}}\right),
$$

and

$$
\frac{D_{i}}{D_{0 i}}=1+B_{i} \sum_{j, l= \pm 1} \cos \left(2 \pi \frac{F_{\beta}+j \Delta F_{\perp}+l \Delta F_{c}}{B_{z}}\right),
$$


The product of Eqs. (B1) and (B2) gives

$$
\begin{aligned}
\frac{\sigma_{i}}{\sigma_{i}^{(0)}}= & 1+\left(A+B_{i}\right) \sum_{j, l= \pm 1} \cos \left(2 \pi \frac{F_{\beta}+j \Delta F_{\perp}+l \Delta F_{c}}{B_{z}}\right) \\
& +A_{\beta} B_{i} \sum_{j, l, j^{\prime}, l^{\prime}= \pm 1} \cos \left(2 \pi \frac{F_{\beta}+j \Delta F_{\perp}+l \Delta F_{c}}{B_{z}}\right) \times \\
& \times \cos \left(2 \pi \frac{F_{\beta}+j j^{\prime} \Delta F_{\perp}+l l^{\prime} \Delta F_{c}}{B_{z}}\right),
\end{aligned}
$$

where $\sigma_{i}^{(0)}=e^{2} g_{0} D_{0 i}$ does not oscillate. The second term in Eq. (B3) gives the first harmonic of MQO with amplitudes $\sim A$ and four close frequencies $F_{\beta} \pm \Delta F_{\perp} \pm \Delta F_{c} \sim$ $F_{\beta} \gg \Delta F_{\perp}$, corresponding to one splitted FS pocket. The last term in Eq. (B3) is of the second order in the amplitude $A_{\beta}$ and gives various frequencies: (i) for $j^{\prime}=l^{\prime}=1$ the 4 second harmonics $2\left(F_{\beta} \pm \Delta F_{\perp} \pm \Delta F_{c}\right)$, which are strongly damped by temperature and disorder and can be neglected; (ii) for $j^{\prime}=1$ and $l^{\prime}=-1$ the SlO with ultra-low frequency $2 \Delta F_{c} \ll \Delta F_{\perp} \ll F_{\beta}$, corresponding to the very low frequency $\sim 100 T$ recently observed $27^{27}$ in YBCO; (iii) for $j^{\prime}=-1$ and $l^{\prime}= \pm 1$ the $\mathrm{SlO}$ with intermediate frequencies $2 \Delta F_{\perp}$ and $2 \Delta F_{\perp} \pm$ $2 \Delta F_{c}$, which correspond to the observed $F_{\alpha} \approx 530 \mathrm{~T}$ and $F_{ \pm}=F_{\alpha} \pm \Delta F_{\alpha}$ frequencies in YBCO. Indeed, using the identity $2 \cos x \cos y=\cos (x-y)+\cos (x+y)$ and neglecting the high frequency $\left(\sim 2 F_{\beta}\right)$ contributions $\cos (x+y)$ we can rewrite the last term in Eq. (B33) for $j^{\prime}=-1$ as

$$
\begin{gathered}
\frac{A_{\beta} B_{i, \beta}}{2} \sum_{j, l, l^{\prime}= \pm 1} \cos \left(2 \pi \frac{2 j \Delta F_{\perp}+l\left(1-l^{\prime}\right) \Delta F_{c}}{B_{z}}\right)= \\
A_{\beta} B_{i, \beta}\left[2 \cos \left(\frac{4 \pi \Delta F_{\perp}}{B_{z}}\right)+\sum_{l= \pm 1} \cos \left(4 \pi \frac{\Delta F_{\perp}+l \Delta F_{c}}{B_{z}}\right)\right] .
\end{gathered}
$$

The first term in the square brackets, corresponding to $l^{\prime}=1$, gives the central peak, while the last term, corresponding to $l^{\prime}=-1$, gives two side peaks. The amplitude of the central peak is doubled because there are four different combinations of $j, l$ giving this term: any $j$ and $l$ at $l^{\prime}=1$. On contrary, each side peak is given by only two combinations of $j, l$ at $l^{\prime}=-1: j=l$ for the right side peak, and $j=-l$ for the left side peak. This combinatoric analysis was proposed in Ref $\underset{59}{ }$.

\section{Appendix C: Possible appearance of slow oscillations in magnetization}

Similarly one can explain the fact that the slow oscillations with frequency $F_{\alpha} \approx 530 T$ are observed also in dHvA effect, which again contradicts the simple model above and that of Refs $\underline{51,52}$. If the electron-electron interaction is included, its effects are roughly proportional to the product of the oscillating density of states (DoS), and it should produce a nonlinearity in the magnetic oscillations of magnetization (or of other thermodynamic quantities) as a functional of the density of states. This nonlinearity results in slow oscillations of thermodynamic quantities such as magnetization, similar to those of the magneto-resistance.

Slow oscillations originate from mixing of different but close fundamental frequencies of magnetic oscillations. This mixing requires some nonlinearity. Transport properties naturally contain such nonlinearity, because they are determined by the density of states and diffusion coefficient. However, the thermodynamic potential

$$
\Omega(\mu, B)=-k_{B} T \int d \varepsilon \rho(\varepsilon, B) \ln \left(1+\exp \frac{\mu-\varepsilon}{k_{B} T}\right),
$$

is a linear functional of the density of states (DoS) $\rho(\varepsilon, B)$. Hence, thermodynamic quantities, e.g. magnetization $M=-\partial \Omega(\mu, B) / \partial B$, are also linear functionals of the density of states (DoS) $\rho(\varepsilon, B) \underline{\underline{67}}$ Hence, for slow oscillations to take place in magnetization, the non-linearity must appear already in the DoS $\rho(\varepsilon, B)$. The DoS is related to the imaginary part of the retarded Green's function $G_{R}(\eta, \varepsilon)$ as $\rho(\varepsilon, B)=$ $-\sum_{\eta} \operatorname{Im} G_{R}(\eta, \varepsilon) / \pi$, where $\eta=\left\{n, k_{y}, k_{z}\right\}$ is a set of quantum numbers of quasiparticles in magnetic field. This Green's function $G_{R}(\eta, \varepsilon)$ includes all relevant types of interaction: with impurities, electron-phonon and electron-electron, which are contained in the self-energy part $\Sigma_{R} \equiv \Sigma_{R}(\eta, \varepsilon, B): G_{R}(\eta, \varepsilon)=\left[\varepsilon-\epsilon(\eta, B)-\Sigma_{R}\right]^{-1}$, where $\epsilon(\eta, B)$ is the bare energy spectrum in the presence of magnetic field. Hence, the DoS

$$
\rho(\varepsilon, B)=\sum_{\eta} \frac{-\operatorname{Im} \Sigma_{R}(\eta, \varepsilon, B) / \pi}{\left[\varepsilon-\epsilon(\eta, B)-\operatorname{Re} \Sigma_{R}(\eta, \varepsilon, B)\right]^{2}+\left[\operatorname{Im} \Sigma_{R}(\eta, \varepsilon, B)\right]^{2}} .
$$

If the self-energy part $\Sigma(\eta, \varepsilon, B)$ is independent of $\eta$, the sum over $\eta$ in Eq. (C2) can be calculated analytically. In quasi-2D metals $\epsilon(\eta, B)=\hbar \omega_{c}(n+\gamma)+2 t_{z} \cos \left(k_{z} d\right)$, and applying the Poisson summation formula one obtains $\frac{52,55}{5}$

$$
\frac{\rho(\varepsilon, B)}{\rho_{0}}=1+2 \sum_{k=1}^{\infty}(-1)^{k} J_{0}\left(\frac{4 \pi k t_{z}}{\hbar \omega_{c}}\right) \exp \left(-2 \pi k \frac{\left|\operatorname{Im} \Sigma_{R}(\varepsilon, B)\right|}{\hbar \omega_{c}}\right) \cos \left(2 \pi k \frac{\varepsilon-\operatorname{Re} \Sigma_{R}(\varepsilon, B)}{\hbar \omega_{c}}\right) .
$$


The non-linearity of the oscillating field dependence is clearly seen from this expression. Naively, one would expect that already the impurity scattering, which in the Born approximation gives oscillating $\operatorname{Im} \Sigma_{R}(\varepsilon, B) \propto$ $\rho(\varepsilon, B)$, results to slow oscillations of $\rho(\varepsilon, B)$. However, this is not the case, because, as first shown in Ref ${ }^{52}$, in the lowest (second) order of the Dingle factor $\exp \left(-2 \pi\left|\operatorname{Im} \Sigma_{R}(\varepsilon, B)\right| / \hbar \omega_{c}\right)$, the contribution from $\operatorname{Re} \Sigma_{R}$ exactly cancels the contribution from $\operatorname{Im} \Sigma_{R}(\varepsilon, B)$ to the slow oscillations. Of course, if one goes beyond the Born approximation and/or includes also the e-e interaction, this exact cancellation does not take place, which results to slow oscillations of magnetization. Therefore, in organic metals, which are rather good clean metals, the slow oscillations of magnetization were not observed. 51 However, in cuprates, where e-e interaction is very strong and the impurity concentration is very high, the slow oscillations of magnetization should appear.
1 A.A. Abrikosov, Fundamentals of the theory of metals, North-Holland, 1988.

2 Shoenberg D. Magnetic oscillations in metals, Cambridge University Press 1984.

3 J. M. Ziman, Principles of the Theory of Solids, Cambridge Univ. Press 1972.

4 Nicolas Doiron-Leyraud, Cyril Proust, David LeBoeuf, Julien Levallois, Jean-Baptiste Bonnemaison, Ruixing Liang, D. A. Bonn, W. N. Hardy, Louis Taillefer, Nature 447, 565 (2007).

5 Suchitra E. Sebastian and Cyril Proust, Annu. Rev. Condens.Matter Phys. 6, 411 (2015) and references therein.

6 S. E. Sebastian, N. Harrison, and G. G. Lonzarich, Rep. Prog. Phys. 75, 102501 (2012).

7 Baptiste Vignolle, David Vignolles, Marc-Henri Julien, and Cyril Proust, Comptes Rendus Phys. 14, 39 (2013).

8 Suchitra E. Sebastian, Neil Harrison and Gilbert G. Lonzarich, Phil. Trans. R. Soc. A 369, 1687 (2011).

9 T. Helm, M. V. Kartsovnik, M. Bartkowiak, N. Bittner, M. Lambacher, A. Erb, J. Wosnitza, and R. Gross, Phys. Rev. Lett. 103, 157002 (2009).

10 T. Helm, M. V. Kartsovnik, I. Sheikin, M. Bartkowiak, F.Wolff-Fabris, N. Bittner, W. Biberacher, M. Lambacher, A. Erb, J. Wosnitza et al., Phys. Rev. Lett. 105, 247002 (2010).

11 M.V. Kartsovnik,T. Helm, C. Putzke, F.Wolff-Fabris, I. Sheikin, S. Lepault, C. Proust, D. Vignolles, N. Bittner,W. Biberacher et al., New J. Phys. 13, 015001 (2011).

12 T. Helm, M. V. Kartsovnik, C. Proust, B. Vignolle, C. Putzke, E. Kampert, I. Sheikin, E.-S. Choi, J. S. Brooks, N. Bittner, W. Biberacher, A. Erb, J. Wosnitza, and R. Gross, Phys. Rev. B 92, 094501 (2015).

13 Taichi Terashima, Nobuyuki Kurita, Megumi Tomita, Kunihiro Kihou, Chul-Ho Lee, Yasuhide Tomioka, Toshimitsu Ito, Akira Iyo, Hiroshi Eisaki, Tian Liang, Masamichi Nakajima, Shigeyuki Ishida, Shin-ichi Uchida, Hisatomo Harima, and Shinya Uji, Phys. Rev. Lett. 107, 176402 (2011).

14 D. Graf, R. Stillwell, T. P. Murphy, J.-H. Park, E. C. Palm, P. Schlottmann, R. D. McDonald, J. G. Analytis, I. R. Fisher, and S. W. Tozer, Phys. Rev. B. 85, 134503 (2012).

15 Amalia I. Coldea, Daniel Braithwaite, Antony Carrington, Comptes Rendus Physique 14, 94 (2013).

16 T. Terashima, N. Kikugawa, A. Kiswandhi, E.-S. Choi, J. S. Brooks, S. Kasahara, T. Watashige, H. Ikeda, T. Shibauchi, Y. Matsuda, T. Wolf, A. E. Böhmer, F. Hardy, C. Meingast, H. v. Löhneysen, M.-T. Suzuki, R. Arita, and S. Uji, Phys. Rev. B. 90, 144517 (2014).

17 A. Audouard, F. Duc, L. Drigo, P. Toulemonde, S. Karlsson, P. Strobel, and A. Sulpice, Europhys. Lett. 109, 27003
(2015).

18 M. D. Watson, T. K. Kim, A. A. Haghighirad, N. R. Davies, A. McCollam, A. Narayanan, S. F. Blake, Y. L. Chen, S. Ghannadzadeh, A. J. Schofield, M. Hoesch, C. Meingast, T. Wolf, and A. I. Coldea, Phys. Rev. B 91, 155106 (2015).

19 M. D. Watson, T. Yamashita, S. Kasahara, W. Knafo, M. Nardone, J. Beard, F. Hardy, A. McCollam, A. Narayanan, S. F. Blake, T. Wolf, A. A. Haghighirad, C. Meingast, A. J. Schofield, H. v. Löhneysen, Y. Matsuda, A. I. Coldea, and T. Shibauchi, Phys. Rev. Lett. 115, 027006 (2015).

20 Suchitra E. Sebastian, N. Harrison, E. Palm, T. P. Murphy, C. H. Mielke, Ruixing Liang, D. A. Bonn, W. N. Hardy and G. G. Lonzarich, Nature 454, 200 (2008)

21 Alain Audouard, Cyril Jaudet, David Vignolles, Ruixing Liang, D. A. Bonn, W. N. Hardy, Louis Taillefer, and Cyril Proust, Phys. Rev. Lett. 103, 157003 (2009).

22 John Singleton, Clarina de la Cruz, R. D. McDonald, Shiliang Li, Moaz Altarawneh, Paul Goddard, Isabel Franke, Dwight Rickel, C. H. Mielke, Xin Yao, and Pengcheng Dai, Phys. Rev. Lett. 104, 086403 (2010).

23 S. E. Sebastian, N. Harrison, M. M. Altarawneh, C. H. Mielke, R. Liang, D. A. Bonn, W. N. Hardy, G. G. Lonzarich, Proc. Natl. Acad. Sci. U.S.A. 107, 6175 (2010).

24 Suchitra E. Sebastian, N. Harrison, P. A. Goddard, M. M. Altarawneh, C. H. Mielke, Ruixing Liang, D. A. Bonn, W. N. Hardy, O. K. Andersen, and G. G. Lonzarich, Phys. Rev. B 81, 214524 (2010).

25 Suchitra E. Sebastian, N. Harrison, Ruixing Liang, D. A. Bonn, W. N. Hardy, C. H. Mielke, and G. G. Lonzarich, Phys. Rev. Lett. 108, 196403 (2012).

26 S.E. Sebastian, N. Harrison, F.F. Balakirev, M.M. Altarawneh, P.A. Goddard et al., Nature 511, 61 (2014).

27 N. Doiron-Leyraud, S. Badoux, S. Rene de Cotret, S. Lepault, D. LeBoeuf, F. Laliberte, E. Hassinger, B.J. Ramshaw, D.A. Bonn, W.N. Hardy,R. Liang, J.-H.. Park, D. Vignolles, B. Vignolle, L. Taillefer and C. Proust, Nature Comm. 6, 6034 (2015).

28 D. Fournier, G. Levy, Y. Pennec, J. L. McChesney, A. Bostwick, E. Rotenberg, R. Liang, W. N. Hardy, D. A. Bonn, I. S. Elfimov \& A. Damascelli, Nature Physics 6, 905 (2010).

29 S. V. Borisenko, A. A. Kordyuk, V. Zabolotnyy, J. Geck, D. Inosov, A. Koitzsch, J. Fink, M. Knupfer, B. Buchner, V. Hinkov, C. T. Lin, B. Keimer, T. Wolf, S. G. Chiuzbaian, L. Patthey, and R. Follath, Phys. Rev. Lett. 96, 117004 (2006).

30 T. Pereg-Barnea, H.Weber, G. Refael and M. Franz, Quantum oscillations from Fermi arcs. Nat. Phys. 6, 44-49 (2010). 
31 G. Ghiringhelli, M. Le Tacon, M. Minola, S. BlancoCanosa, C. Mazzoli, N. Brookes, G. De Luca, A. Frano, D. Hawthorn, F. He et al., Science 337, 821 (2012).

32 J. Chang, E. Blackburn, A. Holmes, N. Christensen, J. Larsen, J. Mesot, R. Liang, D. Bonn, W. Hardy, A. Watenphul et al., Nat. Phys. 8, 871 (2012).

33 A. J. Achkar, R. Sutarto, X. Mao, F. He, A. Frano, S. Blanco-Canosa, M. Le Tacon, G. Ghiringhelli, L. Braicovich, M. Minola, M. Moretti Sala, C. Mazzoli, R. Liang, D. A. Bonn, W. N. Hardy, B. Keimer, G. A. Sawatzky, and D. G. Hawthorn, Phys. Rev. Lett. 109, 167001 (2012).

${ }^{34}$ S. Gerber, H. Jang, H. Nojiri, S. Matsuzawa, H. Yasumura, D. A. Bonn, R. Liang, W. N. Hardy, Z. Islam, A. Mehta, S. Song, M. Sikorski, D. Stefanescu, Y. Feng, S. A. Kivelson, T. P. Devereaux, Z.-X. Shen, C.-C. Kao, W.-S. Lee, D. Zhu, J.-S. Lee, Science 350, 949 (2015); H. Jang, W.-S. Lee, H. Nojiri, S. Matsuzawa, H. Yasumura, L. Nie, A. V. Maharaj, S. Gerber, Y. Liu, A. Mehta, D. A. Bonn, R. Liang, W. N. Hardy, C. A. Burns, Z. Islam, S. Song, J. Hastings, T. P. Devereaux, Z.-X. Shen, S. A. Kivelson, C.-C. Kao, D. Zhu, J.-S. Lee, PNAS 113, 14645 (2016).

35 T. Wu, H. Mayaffre, S. Krämer, M. Horvatic, C. Berthier, W. Hardy, R. Liang, D. Bonn, and M.-H. Julien, Nature (London) 477, 191 (2011).

36 T. Wu, H. Mayaffre, S. Kräamer, M. Horvatic, C. Berthier, W. Hardy, R. Liang, D. Bonn, and M.-H. Julien, Nat. Commun. 6, 6438 (2015).

37 David LeBoeuf, S. Krämer, W. N. Hardy, Ruixing Liang, D. A. Bonn \& Cyril Proust, Nature Physics 9, 79 (2013).

38 David LeBoeuf, Nicolas Doiron-Leyraud, Julien Levallois, R. Daou, J.-B. Bonnemaison, N. E. Hussey, L. Balicas, B. J. Ramshaw, Ruixing Liang, D. A. Bonn, W. N. Hardy, S. Adachi, Cyril Proust \& Louis Taillefer, Nature 450, 533 (2007).

39 S. Badoux, W. Tabis, F. Laliberte, G. Grissonnanche, B. Vignolle, D. Vignolles, J. Beard, D. A. Bonn, W. N. Hardy, R. Liang, N. Doiron-Leyraud, Louis Taillefer \& Cyril Proust, Nature 531, 210 (2016).

40 J. Chang, R. Daou, Cyril Proust, David LeBoeuf, Nicolas Doiron-Leyraud, Francis Laliberte, B. Pingault, B. J. Ramshaw, Ruixing Liang, D. A. Bonn, W. N. Hardy, H. Takagi, A. B. Antunes, I. Sheikin, K. Behnia, and Louis Taillefer, Phys. Rev. Lett. 104, 057005 (2010).

41 I. S. Elfimov, G. A. Sawatzky, and A. Damascelli, Phys. Rev. B 77, 060504(R) (2008).

42 David Garcia-Aldea and Sudip Chakravarty, New Journal of Physics 12, 105005 (2010).

${ }^{43}$ N. Harrison and S. E. Sebastian, New Journal of Physics 14, 095023 (2012).

44 N. Harrison, B. J. Ramshaw and A. Shekhter, Scientific Reports 5, 10914 (2015).

45 A. K. R. Briffa, E. Blackburn, S. M. Hayden, E. A. Yelland, M. W. Long, and E. M. Forgan, Phys. Rev. B 93, 094502 (2016).

46 E. A. Yelland, J. Singleton, C. H. Mielke, N. Harrison, F. F. Balakirev, B. Dabrowski, and J. R. Cooper, Phys. Rev. Lett. 100, 047003 (2008).

47 A. F. Bangura, J. D. Fletcher, A. Carrington, J. Levallois, M. Nardone, B. Vignolle, P. J. Heard, N. Doiron-Leyraud, D. LeBoeuf, L. Taillefer, S. Adachi, C. Proust, and N. E. Hussey, Phys. Rev. Lett. 100, 047004 (2008).

48 B. S. Tan, N. Harrison, Z. Zhu, F. Balakirev, B. J. Ramshaw, A. Srivastava, S. A. Sabok-Sayr, B. Dabrowski,
G. G. Lonzarich, and Suchitra E. Sebastian, Proc. Natl. Acad. Sci. U.S.A. 112, 9568 (2015).

49 Doping changes the size of unreconstructed Fermi-surface considerably, giving a very large relative change of the size of any small reconstructed Fermi-surface pockets.

50 B. J. Ramshaw, S. E. Sebastian, R. D. McDonald, James Day, B. S. Tan, Z. Zhu, J. B. Betts, Ruixing Liang, D. A. Bonn, W. N. Hardy, N. Harrison, Science 348, 317 (2015). See in particular Fig. 2.

51 M.V. Kartsovnik, P.D. Grigoriev, W. Biberacher, N.D. Kushch, P. Wyder, Phys. Rev. Lett. 89, 126802 (2002).

52 P.D. Grigoriev, Phys. Rev. B 67, 144401 (2003).

53 P.D. Grigoriev, A. A. Sinchenko, P. Lejay, A. Hadj-Azzem, J. Balay, O. Leynaud, V. N. Zverev and P. Monceau, Eur. Phys. J. B 89, 151 (2016). In this case slow oscillations in bilayer rare-earth tellurides at a single frequency were interpreted as coming from an interlayer coupling but with no warping.

54 R.B. Dingle, Proc. Roy. Soc. A211, 517 (1952).

55 T. Champel and V. P. Mineev, Phil. Magazine B 81, 55 (2001).

56 P. D. Grigoriev, M. V. Kartsovnik, W. Biberacher, Phys. Rev. B 86, 165125 (2012).

57 Eq. (19) assumes that $t_{z}\left(\boldsymbol{k}_{\|}\right) \approx$ const. When $t_{z}\left(\boldsymbol{k}_{\|}\right)$has a strong angular dependence, Eq. (19) is modified, ${ }^{68-70}$ but remains an oscillating function of $k_{F} c^{\star} \tan \theta$.

${ }^{58}$ K. Yamaji, J. Phys. Soc. Jpn. 58, 1520 (1989).

59 P.D. Grigoriev and T. Ziman, JETP Lett. 106(6), in press (2017) [Pisma v ZhETF 106(6), 349 (2017)].

60 O. K. Andersen, A.I. Liechtenstein, O. Jepsen, F. Paulsen, J. Phys. Chem. Solids 56, 1573 (1995).

61 P.D. Grigoriev and T.I. Mogilyuk, to be published.

${ }^{62}$ We take $m_{\beta}^{*} \approx 3.8 m_{e}$ as obtained from dHvA measurements for $\beta$-frequency, ${ }^{20}$ because within our model $m_{\alpha}^{*} \approx$ $1.6 m_{e}$ is not a true effective electron mass but rather an effective parameter coming from the temperature dependence of the square of Dingle temperature (see Sec.IIC).

63 An excellent fit of experimental data by the temperature damping factor $R_{T}$ of the Lifshitz-Kosevich formula has been achieved (see Fig. 6c in Ref. ${ }^{\frac{5}{}}$ ), at least for one doping level $p \approx 0.108$ over a wide temperature interval $1 K<T<16 \mathrm{~K}$. Such a good fit is atypical even for materials which are much cleaner and have stoichiometric chemical composition and almost ideal crystal structure. In any case, the temperature-dependent Dingle factor due to e-e interactions in YBCO should spoil the ideal dependence $R_{T}(T)$, which in Fig. 6c in Ref. ${ }^{\frac{5}{-}}$ survived even up to $T=16 \mathrm{~K}$. Therefore, we believe that the quality of the fit may be accidental.

${ }^{64}$ The electron level broadening $\Gamma$ is related to the Dingle temperature $T_{D}=\Gamma / k_{B}$ and to the effective electron mean free time $\tau=\hbar / 2 \Gamma$. Usually, at low temperatures one takes into account only electron scattering by impurities, which gives a temperature-independent Dingle factor. The contribution to $T_{D}$ from electron-electron (e-e) and electronphonon (e-ph) increases with temperature and may be confused with the damping factor coming from temperature $R_{T}$, thus leading to the incorrect estimate of the effective mass from the oscillations. While in the lowest order of perturbation theory the electron-phonon contributions to $T_{D}$ and to $m^{*}$ were shown ${ }^{2,65}$ to compensate each other, the electron-electron contribution to $T_{D}$ in disordered metals needs further theoretical study. 
65 S. Engelsberg, G. Simpson, Phys. Rev. B 2, 1657 (1970).

66 Y. Adamov, I. V. Gornyi, and A. D. Mirlin, Phys. Rev. B 73, 045426 (2006).

${ }^{67}$ For simplicity we assume the chemical potential $\mu$ to be constant. In the opposite limit of constant particle density the chemical potential is an oscillating function of magnetic field,,$\frac{71,72}{1}$ which gives additional nonlinearity to the functional $M[\rho(\varepsilon, B)]$.

${ }^{68}$ M. V. Kartsovnik, V. N. Laukhin, S. I. Pesotskii, I. F.
Schegolev, V. M. Yakovenko, J. Phys. I 2, 89 (1992).

69 C. Bergemann, S. R. Julian, A. P. Mackenzie, S. NishiZaki, and Y. Maeno, Phys. Rev. Lett. 84, 2662 (2000).

70 P.D. Grigoriev, Phys. Rev. B 81, 205122 (2010).

71 P. Grigoriev, JETP 92, 1090 (2001) [Zh. Eksp. Teor. Fiz. 119(6), 1257 (2001)].

72 Thierry Champel, Phys. Rev. B 64, 054407 (2001). 